\title{
Correction to: Effect of salicylic acid foliar application on growth, glandular hairs and essential oil yield in Salvia officinalis L. grown under zinc stress
}

\author{
Fatima Zohra Es-sbihi ${ }^{*}$, Zakaria Hazzoumi ${ }^{2}$ and Khalid Amrani Joutei ${ }^{1}$
}

\section{Correction to: Chem Biol Technol Agric (2020) 7:26}

https://doi.org/10.1186/s40538-020-00192-6

Following the publication of the original article [1], the authors would like to correct the error in the materials and methods section under the heading $\mathbf{Z n}$ and SA treatments.

The sentence currently reads:

This experiment includes total six treatments: control, $0.5 \mathrm{mM}$ salicylic acid (SA $0.5 \mathrm{mM}), 1 \mathrm{mM}$ salicylic acid (SA $1 \mathrm{mM}), 40 \mathrm{mM} \mathrm{ZnSO} 4(\mathrm{Cu}), 40 \mathrm{mM}$ $\mathrm{ZnSO} 4+0.5 \mathrm{mM}$ salicylic acid $(\mathrm{Zn}+\mathrm{SA} 0.5 \mathrm{mM})$ and $40 \mathrm{mM} \mathrm{ZnSO} 4+1 \mathrm{mM}$ salicylic acid $(\mathrm{Zn}+\mathrm{SA} 1 \mathrm{mM})$.

The sentence should read:

This experiment includes total six treatments: control, $0.5 \mathrm{mM}$ salicylic acid (SA $0.5 \mathrm{mM}), 1 \mathrm{mM}$ salicylic acid (SA $1 \mathrm{mM}$ ), $40 \mathrm{mM}$ ZnSO4 (Zn), $40 \mathrm{mM}$ $\mathrm{ZnSO} 4+0.5 \mathrm{mM}$ salicylic acid $(\mathrm{Zn}+\mathrm{SA} 0.5 \mathrm{mM})$ and $40 \mathrm{mM} \mathrm{ZnSO} 4+1 \mathrm{mM}$ salicylic acid (Zn + SA $1 \mathrm{mM})$.

The author group has been updated above and the original article [1] has been corrected.

\begin{abstract}
Author details
${ }^{1}$ Microbial Biotechnology and Bioactive Molecules Laboratory, Faculty of Science and Technology Fez, Sidi Mohamed Ben Abdellah University, B.P. 2202, Road of Imouzzer, Fez, Morocco. ${ }^{2}$ AgroBioSciences Mohammed VI Poly-technic University, Lot 660, Hay Moulay Rachid, 43150 Benguerir, Morocco.
\end{abstract}

Published online: 05 February 2021

\section{Reference}

1. Es-sbihi FZ, Hazzoumi Z, Joutei KA. Effect of salicylic acid foliar application on growth, glandular hairs and essential oil yield in Salvia officinalis L. grown under zinc stress. Chem Biol Technol Agric. 2020;7:26. https://doi. org/10.1186/s40538-020-00192-6.

\section{Publisher's Note}

Springer Nature remains neutral with regard to jurisdictional claims in published maps and institutional affiliations. 